OPEN ACCESS

Edited by:

Alberto Farolfi,

Romagnolo Scientific Institute for the

Study and Treatment of Tumors

(IRCCS), Italy

Reviewed by:

Martina Arcieri,

University of Eastern Piedmont, Italy Xiaoping Wan

Shanghai First Maternity and Infant

Hospital, China

*Correspondence: Ling Ouyang

ouyl@sj-hospital.org

Specialty section:

This article was submitted to

Women's Cancer,

a section of the journal

Frontiers in Oncology

Received: 12 January 2020

Accepted: 01 May 2020

Published: 11 June 2020

Citation:

Liu D, Song Z, Wang X and Ouyang L (2020) Ubiquitin C-Terminal Hydrolase L5 (UCHL5) Accelerates the Growth of Endometrial Cancer via Activating the Wnt/ $\beta$-Catenin Signaling Pathway.

Front. Oncol. 10:865

doi: 10.3389/fonc.2020.00865

\section{Ubiquitin C-Terminal Hydrolase L5 (UCHL5) Accelerates the Growth of Endometrial Cancer via Activating the Wnt/ $\beta$-Catenin Signaling Pathway}

\author{
Da Liu, Zixuan Song, Xiaoying Wang and Ling Ouyang* \\ Department of Obstetrics and Gynecology, Shengjing Hospital of China Medical University, Shenyang, China
}

Endometrial cancer $(E C)$ is the most prevalent gynecological malignancy with high mortality. Chemotherapy plays a pivotal role both in an adjuvant setting and in exclusive treatment. However, current pharmacotherapies are limited and not ideal for improving the overall survival of EC patients. Thus, identification of the underlying molecular mechanisms responsible for initiation and progression of EC is imperative for developing novel therapeutic strategies. Ubiquitin C-terminal hydrolase L5 (UCHL5) has been found to aggravate tumor growth and metastasis in several different types of tumor models such as esophageal squamous cell carcinoma, hepatocellular carcinoma, and epithelial ovarian cancer. However, whether UCHL5 influences the growth of EC has not been elucidated. To expose the role of UCHL5 on EC, bioinformatics analysis was conducted, and it hinted that UCHL5 was overexpressed in EC tissues and associated with lower overall survival. Consistently, the overexpression of UCHL5 in EC tissues and cell lines was further confirmed by western blot (WB) and polymerase chain reaction (PCR) compared with non-tumor control. Lentivirus vectors carrying UCHL5 shRNA or CD sequences were used to reduce or overexpress the UCHL5 gene, respectively. Cell proliferation and cycle were facilitated, and cell apoptosis was decreased when the UCHL5 gene was overexpressed in EC cell lines. These results were opposite in UCHL5 knockdown EC cells. Additionally, the expression of $\beta$-catenin is positively related to UCHL5 levels and the tumorigenic effects of UCHL5 overexpression were reversed by the Wnt/ $\beta$-catenin pathway inhibitor XAV939. Thus, Wnt/ $\beta$-catenin pathway activation may be a partial mechanism responsible for the promoting effects of UCHL5 on EC growth. In conclusion, UCHL5 accelerated the growth of EC via the Wnt/ $\beta$-catenin pathway and was expected to be an attractive target for EC treatment.

Keywords: endometrial cancer, UCHL5, lentivirus vectors, Wnt/ $\beta$-catenin pathway, XAV939

\section{INTRODUCTION}

Endometrial cancer (EC) is the most prevalent invasive gynecological malignancy in the western world and the fourth common cancer in women worldwide, with over 280,000 new cases worldwide in 2017 (1). The morbidity is still climbing due to the prevalence of global obesity, the coming of aging society, environmental degradation, etc. (2). Although most patients are diagnosed early and accepted comprehensive treatments, the number of estimated deaths caused by EC was almost 
36,000 annually, accounting for $1.8 \%$ of all cancer deaths (3). More notably, if without follow-up adjuvant therapies such as chemotherapy or radiotherapy, $10-15 \%$ surgical patients will relapse (4). These recurrent cases have a poor prognosis with a 5-year survival rate less than $15 \%$ because of the limited treatment options and resistance to current medications $(4,5)$. Therefore, deeper molecular mechanisms and targeted drugs deserve further exploration.

The ubiquitin-proteasome system (UPS) is known as a specific and selective pathway for most protein degradation in all eukaryotic cells (6). Ubiquitin (Ub), a small 76-residue protein, marks these proteins who are destined for degradation in the form of polymeric chains so that they were accurately recognized and degraded by the proteasome (7). The process of ubiquitination can be reversibly regulated through lengthening or cleaving the poly-Ub chains mediated by ubiquitinating and deubiquitinating enzymes (DUBs), respectively $(7,8)$. Previous investigations have demonstrated the UPS involved in various tumor-promoting processes, including DNA repair, apoptosis, cell cycle, and oncogenic signaling (9). Not surprisingly, the ubiquitination pathway has been considered as a fascinating target for anti-cancer drug research, especially after two proteasome inhibitor drugs (bortezomib and carfilzomib) are successfully approved for treatment of relapsed or refractory multiple myelomas (10). However, the actions of each component in the ubiquitination pathway on some solid tumors, especially $\mathrm{EC}$, remain unclear.

According to the homology and the functional mechanism, DUBs are mainly segmented into five categories: Ub C-terminal hydrolase (UCH), Ub specific protease (USP), ovarian tumor protease (OTU), Josephin/Machado-Joseph disease protease (MJD), and JAB1/MPN/MOV34 metalloenzyme (JAMM) (11). Among 80 cloned DUBs in the human genome, nearly half of them turned out to be associated with the development of various types of cancer, previously (12). Ubiquitin C-terminal hydrolase L5 (UCHL5)/Uch37, a cysteine protease from the family of ubiquitin C-terminal hydrolases (UCHs), can remove $\mathrm{Ub}$ from the distal part of the poly- $\mathrm{Ub}$ chains and rescues poorly ubiquitinated proteins from proteolysis (13). Disabling the UCHL5 gene in mice results in embryonic death, indicating that UCHL5 function is very crucial (14). Overexpression of UHCL5 has been reported in several solid tumors and correlates with the poor survival and increased risk of cancer recurrence (15). Additionally, bAP15, a depressor of the 19S proteasome DUBs (UCHL5 and USP14), exhibits significant inhibition of cell growth and invasion in several human cancers (16). In conclusion, these researches indicated that UCHL5 plays a momentous role in the development of tumors.

The Wnt/ $\beta$-catenin pathway controls various cellular processes of cancer biology, such as cell proliferation, differentiation, and maintenance of pluripotency (17). The function of the Wnt/ $\beta$-catenin pathway on EC growth had been well-established (18). $\beta$-Catenin maintains epithelial cell integrity, an important barrier for blocking tumor metastasis (19). Additionally, hypophosphorylated $\beta$-catenin transfers into the nucleus and binds to transcription factors, leading to transcriptional activation of specific target genes, including
Cyclin D1, C-MYC, and MMP-7, all of which contribute to cell survival, cell cycle, uncontrolled proliferation, and distant metastasis (20).

However, the link between UCHL5 and the Wnt/ $\beta$-catenin pathway and the function of UCHL5 on EC growth have not yet been completely elucidated so far. In this study, we mainly want to solve the following questions: (1) Whether the expression of UCHL5 is significantly different between EC and normal endometrium; (2) How about the effects of UCHL5 on the growth and survival of EC; and (3) Whether the Wnt/ $\beta$-catenin pathway can partly explain the effects of UCHL5 on EC. Our study may contribute to unravel the mechanisms underlying the occurrence and progression of EC and provide new therapeutic targets.

\section{METHODS AND MATERIALS}

\section{EC Cancer and Adjacent Tissue Collection}

All the EC and adjacent non-neoplastic endometrial tissues were obtained from hysterectomy patients diagnosed as EC at the Department of Obstetrics and Gynecology, Shengjing Hospital affiliated with China Medical University, from 2016 to 2017. The EC tissues $(n=10)$ and tumor adjacent tissues $(n=10)$ were separated from excised uteruses and then rapidly frozen with liquid nitrogen and stored at $-80^{\circ} \mathrm{C}$. All patients voluntarily signed an informed consent to donate their excised organ. Additionally, all patients had not received any chemotherapy or radiotherapy before their surgery. Besides, all tissue specimens were reviewed by a pathologist to confirm the diagnosis. This study was approved by the Ethics Committee of the Shengjing Hospital affiliated with China Medical University.

\section{Cell Lines and Cell Culture}

Human endometrial cancer cell AN3-CA (ATCCHTB-111) was purchased from the American Type Culture Collection (ATCC). Other endometrial cancer cells HCE-1-A (BNCC338711), HCE1-B (BNCC100172), Ishikawa (BNCC338359), and human endometrial epithelial cell hEEC (BNCC341726) were purchased from BeNa Culture Collection (BNCC). Each cell was cultured in a basic medium recommended by their user manual, supplemented with $10 \%$ fetal bovine serum (FBS) (GIBCO $\mathrm{BRL}, \mathrm{BRA}, \mathrm{USA})$ and $100 \mathrm{U} / \mathrm{mL}$ penicillin and $100 \mu \mathrm{g} / \mathrm{mL}$ streptomycin (Beyotime Biotechnology, SH, China). Cell culture was carried out at $37^{\circ} \mathrm{C}, 5 \% \mathrm{CO}_{2}$, and $95 \%$ humidity, and cell passage was digested by $0.25 \%(\mathrm{w} / \mathrm{v})$ trypsin containing $0.53 \mathrm{mM}$ EDTA (20).

\section{Lentiviral Vector Construction and Cell Transfection}

Lentiviral-mediated shRNA interference technology was used to downregulate the UCHL5 gene in HCE-1-A cells. Three pairs of shRNA oligonucleotides were designed by GeneChem Company (Gene, SH, China) based on the sequence of the UCHL5 gene (NM_015984.5) and listed in Table 1. Each nucleotide sequence was inserted into the lentivirus core plasmid pLKO.1 (Addgene) after being digested by Agel I/EcoR I at $37^{\circ} \mathrm{C}$. For lentiviral production, the recombinant plasmid described above 
TABLE 1 | Sequences of shUCHL5.

\begin{tabular}{ll}
\hline shUCHL5 & Sequence $\left(\mathbf{5}^{\prime} \mathbf{-} \mathbf{3}^{\prime} \mathbf{)}\right.$ \\
\hline shUCHL5-F1 & CCGGTCCGAGCTCATTAAAGGATTCTCGAGAATCCTTAA \\
& TGAGCTCGGTITG \\
shUCHL5-R1 & AATTCAAAACCGAGCTCATTAAAGGATTCTCGAGAATCC \\
& TTAATGAGCTCGGA \\
shUCHL5-F2 & CCGGTCCGATTGATTAGGTGCATCTCGAGATGCACCTAA \\
& ATCAATCGGTITTG \\
shUCHL5-R2 & AATTCAAAACCGATTGATTAGGTGCATCTCGAGATGC \\
& ACCTAAATCAATCGGA \\
shUCHL5-F3 & CCGGTGCAGAAGATAGCAGAGTACTCGAGTAACTCTGC \\
& TATCTTCTGCTIITG \\
shUCHL5-R3 & AATTCAAAAAGCAGAAGATAGCAGAGTTACTCGAGTA \\
& ACTCTGCTATCTTCTGCA
\end{tabular}

TABLE 2 | Sequences of UCHL5 CDs region.

\begin{tabular}{ll}
\hline oeUCHL5 & Sequence $\left(\mathbf{5}^{\prime} \mathbf{-} \mathbf{3}^{\prime}\right)$ \\
\hline UCHL5-F & CGGAATTCATGACGGGCAATGCCG \\
UCHL5-R & CGGGATCCTCATTTGGTTCCTGAGCTTC \\
\hline
\end{tabular}

and two packaging plasmids (psPAX2 and pMD2G) were cotransfected into HEK293T cells (ATCC) using Lipofectamine 2000 (Invitrogen) according to the manufacturer's instruction described previously (21). Forty-eight to seventy-two hours later, the virus supernatant was harvested and concentrated by ultracentrifugation $\left(4,000 \mathrm{~g}\right.$ at $\left.4^{\circ} \mathrm{C}\right)$ for $10 \mathrm{~min}$.

We used the lentiviral vector system to establish a human endometrial cancer AN3-CA cell stably overexpressing UCHL5 (oeUCHL5). The complete coding sequences (CDs) of UCHL5 were amplified by the primers listed in Table 2 and inserted into the core plasmid pLVX-Puro (Clontech, Laboratories, Inc.) after being digested by EcoR I/BamH I at $37^{\circ} \mathrm{C}$. The recombinant lentiviral vector encoding the UCHL5 gene was produced according to the protocol described above.

For cell infection, cells were planted at a density of 50,000 cells per well in six-well-plates and then, respectively, transduced with purified lentiviruses at a multiplicity of infection of 40 (22). UCHL5 knockdown HCE-1-A cells were established by lentiviral infection containing UCHL5 shRNA sequences (shUCHL5) and UCHL5 overexpression AN3-CA cells were established by lentiviral infection containing UCHL5 CDs. Twenty-four hours after transduction, the medium was replaced and cells were incubated in a $\mathrm{CO}_{2}$ incubator at $37^{\circ} \mathrm{C}$ for later experiments.

\section{Bioinformatics Analysis}

Public data of UCLH5 gene expression in EC patients were acquired from the Cancer Genome Atlas Project (TCGA; http:// cancergenome.nih.gov/). The differences of UCLH5 gene mRNA expression levels were analyzed between 548 EC and 35 nontumor specimens. The overall survival of EC patients with high and low UCLH5 expression was also obtained from the TCGA database. We used the gene set enrichment analysis (GSEA) approach to analyze the effects of UCLH5 differential gene expression on biological annotation and pathways. Enrichment results should satisfy $P<0.05$ with FDR $q<0.25$ in the present study.

\section{Cell Viability Assay}

The cells were planted in 96-well-plates, and the final cell density was adjusted to $3 \times 10^{3}$ /well with fresh medium for $12,24,48$, and $72 \mathrm{~h}$ after lentiviral infection. Cell Counting Kit-8 (Beyotime Biotechnology, SH, China) was used to analyze the cell viability according to the previous description (20).

\section{Cell Apoptosis}

For cell apoptosis, UCHL5 knockdown HCE-1-A cells and UCHL5 overexpression AN3-CA cells treated with or without XAV939 (HY-15147, MedChemExpress, New Jersey, USA), a Wnt/ $\beta$-catenin pathway inhibitor, were collected and then incubated with Annexin V-FITC and Propidium Iodide (PI) in a cassette according to the introduction of Apoptosis Detection Kit (C1062, Beyotime) (23). These labeled cells were directly delivered to a flow cytometer (Becton-Dickinson, Franklin Lakes, NJ, USA) with CellQuest software (BD Biosciences) to analyze the proportion of apoptotic cells without washing.

\section{Cell Cycle Analysis}

For cell cycle analysis, pretreated cells were harvested and fixed by ice-cold $70 \%$ ethanol for $2 \mathrm{~h}$ at $4^{\circ} \mathrm{C}$. Then, cells were incubated by RNase and PI reagent in the darkness according to the Cell Cycle Analysis Kit (C1052, Beyotime) protocol (20). After a 30min incubation, cell cycle was analyzed by a flow cytometer (NovoCyte, Aceabio, San Diego, CA).

\section{Western Blot (WB)}

After being washed with phosphate-buffered saline (PBS), patient tissue specimens or pretreated cells were lysed in RIPA lysis buffer (Beyotime) containing 1\% protease inhibitor (Beyotime). All samples were centrifuged at $16,000 \times \mathrm{g}$ for $20 \mathrm{~min}$ at $4^{\circ} \mathrm{C}$, and then the supernatant was used for protein quantification by BCA protein assay kit (Beyotime). Then, proteins that underwent thermal denaturation at $98^{\circ} \mathrm{C}$ for $10 \mathrm{~min}$ were separated by SDS-PAGE electrophoresis and transferred to polyvinylidene difluoride (PVDF) membranes (Millipore). Subsequently, 5\% non-fat milk in Tris-buffered saline containing $0.1 \%$ Tween 20 (TBST) was used to block the membranes for $1 \mathrm{~h}$ on a rolling device at room temperature. Removing blocking solution, corresponding primary antibodies were added and incubated overnight at $4^{\circ} \mathrm{C}$. Then, these membranes were incubated with horseradish peroxidase (HRP)-conjugated secondary antibodies for another $2 \mathrm{~h}$ at room temperature after being washed six times with TBST buffer. The information of antibodies was below: Survivin (Ab469, Abcam, 1:5,000 dilution), $\beta$-catenin (Ab32572, Abcam, 1:5,000 dilution), UCHL5 (11527-1-AP, Proteintech, 1:500 dilution), CyclinD1 (Ab16663, Abcam, 1:200 dilution), Cleaved-Caspase3 (Ab4051, Abcam, 1:200), C-myc (Ab185656, Abcam, 1:2,000 dilution), Goat anti-mouse (A0216, Beyotime, 1:1,000 dilution), and Goat anti-rabbit (A0208, Beyotime, 1:1,000 dilution) HRP-labeled secondary antibody. GAPDH (60004-1$1 \mathrm{G}$, Proteintech, 1:5,000 dilution) was used as an internal control 
in this study. The relative intensity of proteins was analyzed with Image Lab software (Bio-Rad) after interacting with enhanced chemiluminescence (ECL) chromogenic substrate (Beyotime).

\section{Quantitative Real-Time PCR (qRT-PCR)}

For analysis of relative mRNA level, total RNA extraction from cells or tumors was performed using TRIzol reagent (Invitrogen, Carlsbad, CA, USA), and then reverse-transcribed into cDNA using the M-MLV First-Strand cDNA Synthesis Kit (Invitrogen) (23). The mRNA levels of the UCHL5 gene were quantified by the qRT-PCR method with Power SYBR Green PCR Master Mix (Invitrogen). The primers are listed in Table 3. An Applied Biosystems 7500 Fast Dx Real-Time PCR instrument (Thermo Fisher, USA) was used in the study. GAPDH was taken as an internal control and the results were analyzed using the $2^{-\Delta \Delta \mathrm{Ct}}$ method.

\section{Animal Model}

HCE-1-A cells with or without shUCHL5 lentiviral infection were separated by $0.25 \%$ trypsin, and the concentration of the cell suspension was modulated to $5 \times 10^{6}$ cells $/ 100 \mu \mathrm{L}$, then $100 \mu \mathrm{L}$ cell suspension was injected into the left lower lateral subcutaneous tissue of the nude mice (20). Mice were considered tumor bearing when tumors became palpable at 12 days after the first injection and included into this study. Tumor growth was measured with a caliper using the formula $V=W^{2} L \pi / 6$, where $V$ represents tumor volume, $W$ means short diameter, and $L$ is the mean long diameter. The volume of the xenografts was measured and recorded every 3 days, and the mice were euthanized for 33 days with the tumors separated and weighed.

\section{Statistical Analysis}

Data are presented as means \pm SEM and statistically analyzed by GraphPad Prism 4.0 software (GraphPad Software). The numbers of repetitions and groups about each experimental section are listed in the figure legends. Statistical analysis was based on Student's $t$-test or a one-way ANOVA with Tukey's post-hoc test according to fact conditions. For all tests, a $P$-value of $<0.05$ was considered significant.

\section{RESULTS}

\section{The Expression of UCHL5 Was Significantly Increased in EC Tissues}

In order to find out the levels of UCHL5 expression in EC samples, we first analyzed UCHL5 mRNA expression by comparing $548 \mathrm{EC}$ tissues and 35 normal endometrial tissues from the TCGA database. As shown in Figure 1A, the relative

TABLE 3 | Primer of RT-PCR.

\begin{tabular}{ll}
\hline Genes & Sequence $\left(5^{\prime}-3^{\prime}\right)$ \\
\hline UCHL5-F & ATGAAAGGCTTGGCACTG \\
UCHL5-R & CTCCCATTAACAGGAACATAAC \\
GAPDH-F & GGATTGTCTGGCAGTAGCC \\
GAPDH-R & ATTGTGAAAGGCAGGGAG
\end{tabular}

UCHL5 expression level significantly increased in EC tissues compared with non-tumor specimens. Overall survival (OS) of patients with high UCHL5 mRNA levels and low UCHL5 levels was compared using the TCGA database. The results suggested that EC patients with low UCHL5 mRNA levels had markedly higher OS than those with high UCHL5 mRNA levels (Figure 1B). To analyze the potential biological functions of UCHL5, gene set enrichment analysis (GSEA) was conducted as previously reported (24). Among them, GSEA enrichment plots showed that enrichment of CTNNB1 (the coding gene for $\beta$-catenin) was associated with UCHL5 gene (Figure 1C). Consistent with the bioinformatics results, UCHL5 protein detected by WB was overexpressed in EC tissues compared with matched adjacent normal tissues (Figure 1D).

\section{UCHL5 Richly Expressed in Endometrial Cancer Cell Lines}

Based on the aforementioned results, four human EC cell lines (HCE-1-A, HCE-1-B, AN3-CA, Ishikama) and one human endometrial epithelial cell hEEC were selected to test the UCHL5 expression by qRT-PCR and WB. As shown in Figure 2A, the levels of UCHL5 mRNA were higher in four EC cell lines than those in hEEC cells. Among four human EC cell lines, its expression was highest in HCE-1-A cells and lowest in AN3CA cells. Consistent with RT-PCR results, UCHL5 proteins were higher in EC cell lines to various extents than those in human endometrial epithelial cells (Figures 2B,C). The cell lines with higher expression of UCHL5 mRNA also presented higher expression of UCHL5 protein, suggesting that UCHL5 mRNA stably existed in EC cells and was translated into protein.

\section{UCHL5 Knockdown Inhibited the Growth of Endometrial Cancer Cells}

Since the expression of UCHL5 was highest in HCE-1A endometrial cancer cells, we knocked down UCHL5 expression using lentiviral-mediated shRNA in HCE-1-A cells to demonstrate the actions of UCHL5 on tumorigenesis. All three shRNA sequences significantly reduced the expression of UCHL5 in protein and mRNA levels in HCE-1-A cells (Figures 3A,B). UCHL5 knockdown descended the relative viability of HCE-1-A cells measured by CCK- 8 kits (Figure 3C) and augmented the percentage of apoptosis cells (Figure 3D). Furthermore, we performed flow cytometry to examine the cell cycle distribution after treatment. As shown in Figure 3E, UCHL5 silent cells showed a remarkable increase in the sub-G1 population compared to negative control, indicating that UCHL5 deficiency induced cell cycle arrest.

\section{Upregulation of UCHL5 Promoted the Growth of Endometrial Cancer Cells}

Since the expression of UCHL5 was relatively lower in AN3-CA endometrial cancer cells, we increased the expression of UCHL5 by lentiviral infection in AN3-CA cells. By qRT-PCR and WB analysis, we demonstrated that UCHL5 gene delivery was very efficient (Figures 4A,B). UCHL5 overexpression raised cell viability at different time points after lentiviral infection 

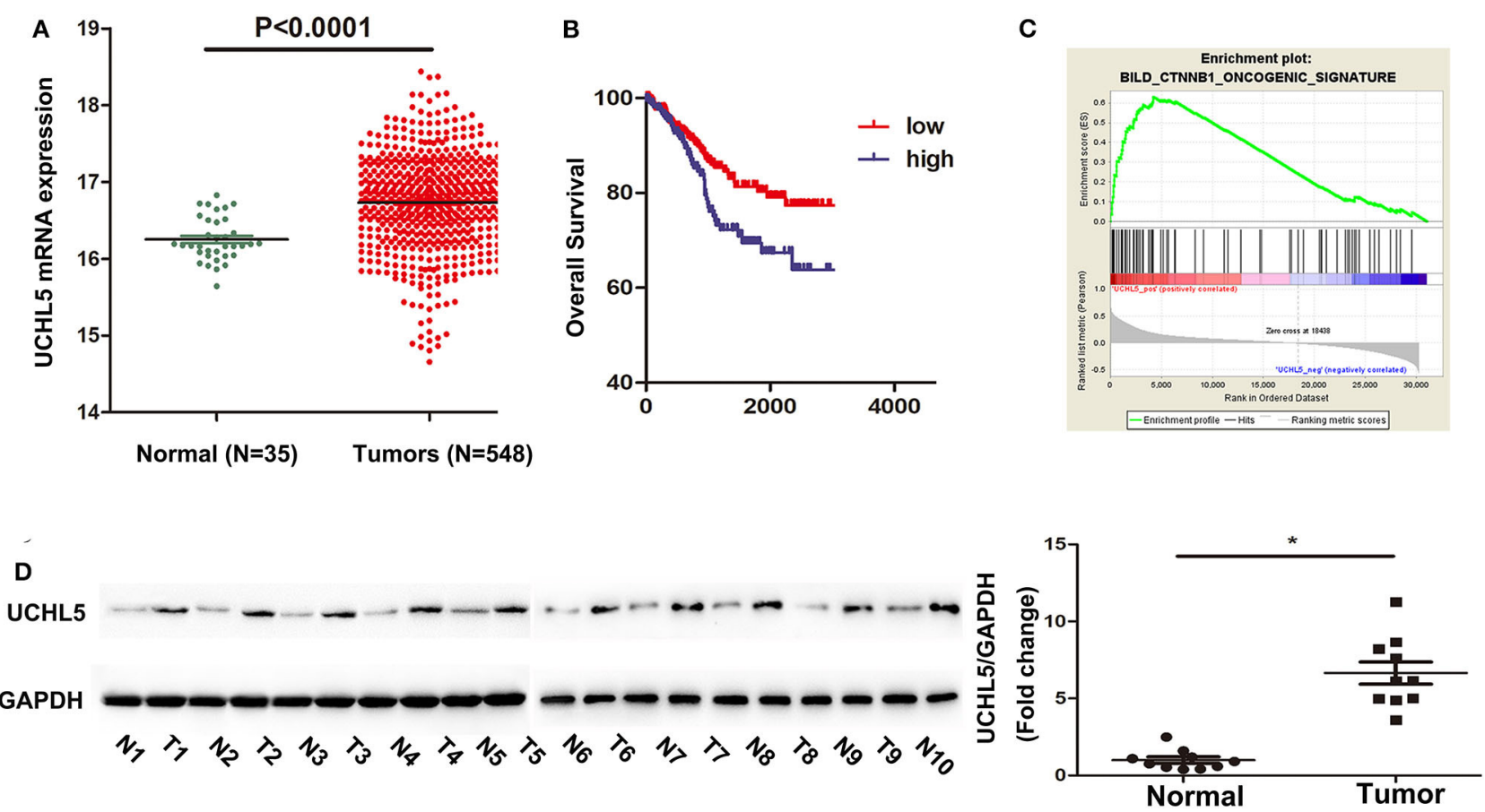

FIGURE 1 | The expression of UCHL5 was significantly increased in EC tissues and negatively correlated with overall survival. (A) The relative levels of UCHL5 mRNA expression between EC tissues $(N=548)$ and non-tumor specimens ( $N=35)$ were obtained from the TCGA database. (B) Overall survival (OS) was analyzed between patients with high UCHL5 and low UCHL5 expression in the TCGA website. (C) Representative image of gene set enrichment plots. (D) Western blot detected the expression of UCHL5 protein between 10 EC tissues (abbreviated to "T") and matched adjacent normal tissues (abbreviated to " $N$ ") ( $N=10)$. $P<0.05$ vs. matched adjacent normal tissues.
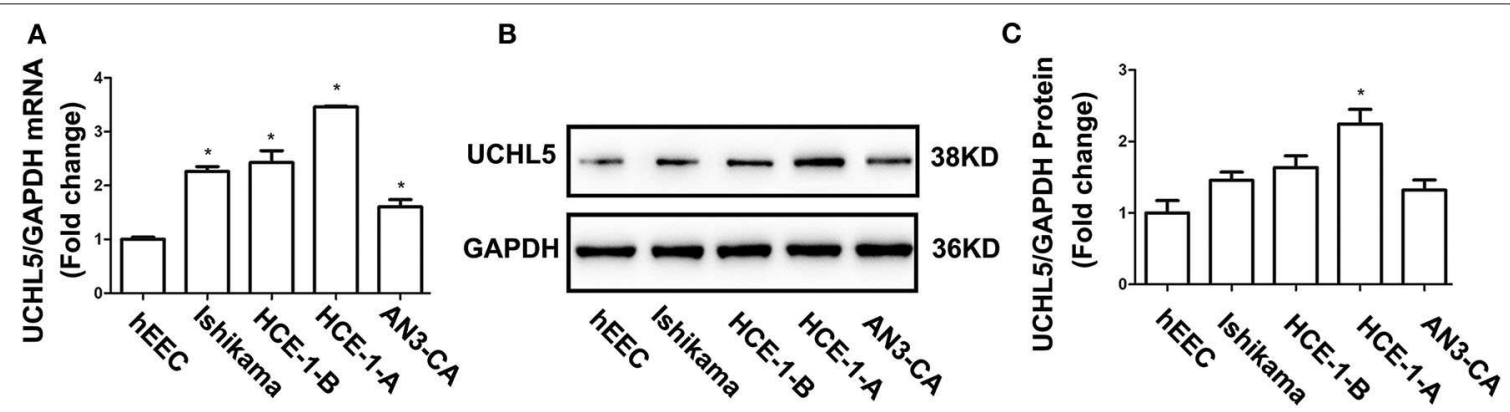

FIGURE 2 | UCHL5 expressed in different endometrial cancer cell lines. (A) Relative mRNA levels of UCHL5 measured by qRT-PCR in human endometrial epithelial cell hEEC and four endometrial cancer cells (Ishikawa, HCE-1-B, HCE-1-A, AN3-CA) ( $N=3$ ). ${ }^{*} P<0.05$ vs. hEEC. (B) Representative bands of UCHL5 and GAPDH in tumors were evaluated by western blot. (C) Statistical diagram of protein levels of UCHL5 ( $N=3$ times). ${ }^{\star} P<0.05$ vs. hEEC.

(Figure 4C). Moreover, UCHL5 overexpression decreased the percentage of apoptosis cells and sub-G1 population (Figures 4D,E). These results indicated that upregulation of UCHL5 expression contributed to the growth of endometrial cancer cells.

\section{UCHL5 Activated Wnt/ $\beta$-Catenin Signaling and Affected the Expression of Its Target Genes}

Retrospecting the bioinformatics results that UCHL5 high expression positively correlated with $\mathrm{Wnt} / \beta$-catenin signaling, we used western blot to detect the influence of UCHL5 on $\beta$-catenin expression, a core element of the $\mathrm{Wnt} / \beta$ catenin signaling pathway. As shown in Figure 5A, UCHL5 knockdown decreased the expression of $\beta$-catenin. CyclinD1 and $\mathrm{C}$-myc and anti-apoptosis protein Survivin were significantly suppressed, but apoptosis-related protein cleaved-caspase 3 was increased in the UCHL5 knockdown HCE-1-A cells compared with negative controls (Figures 5B,C). In contrast, UCHL5 overexpression in AN3-CA cells elevated the expression of $\beta$-catenin and its effector proteins (CyclinD1, C-myc, Survivin) and decreased cleaved-caspase 3 (Figures 5D-F). Taking all these into consideration, UCHL5 possibly regulated cell cyclin, proliferation, and apoptosis via the $\mathrm{Wnt} / \beta$-catenin signaling pathway in EC cells. 
A
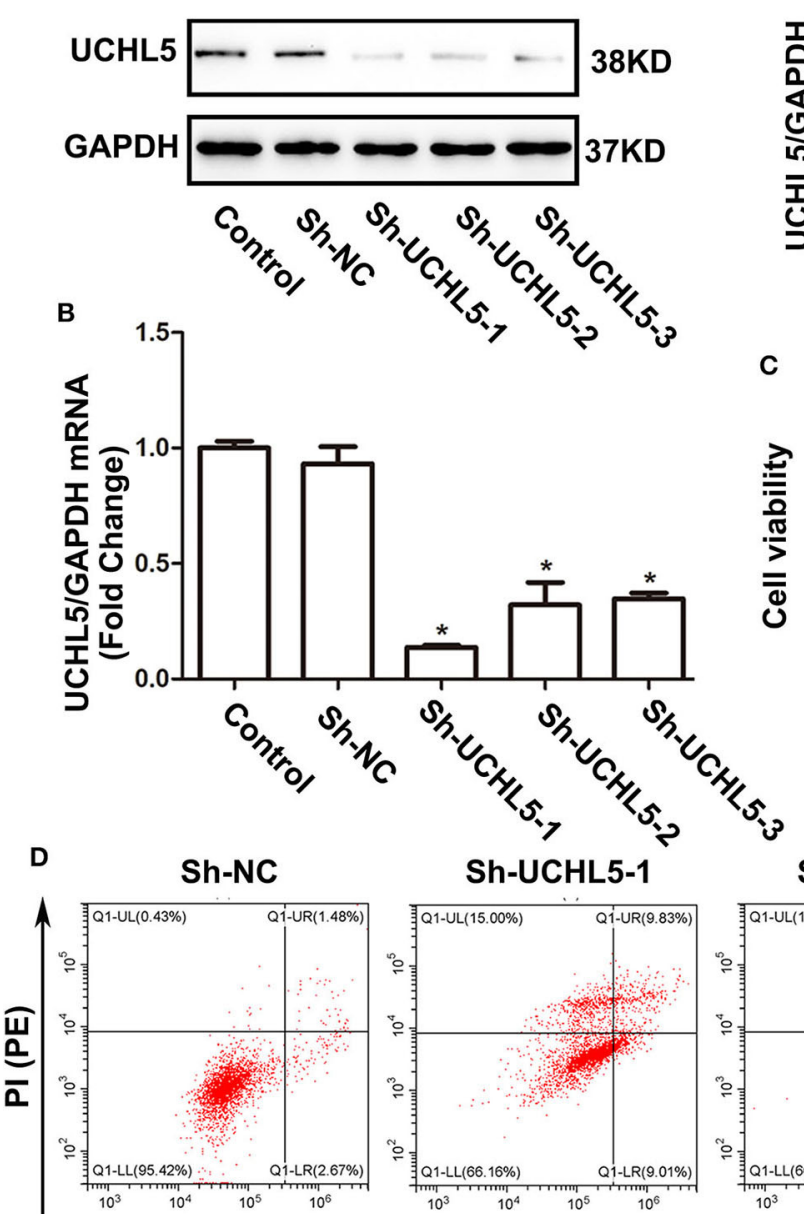

Sh-UCHL5-1

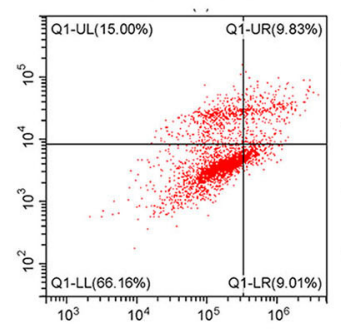

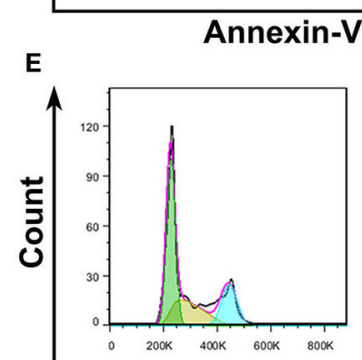
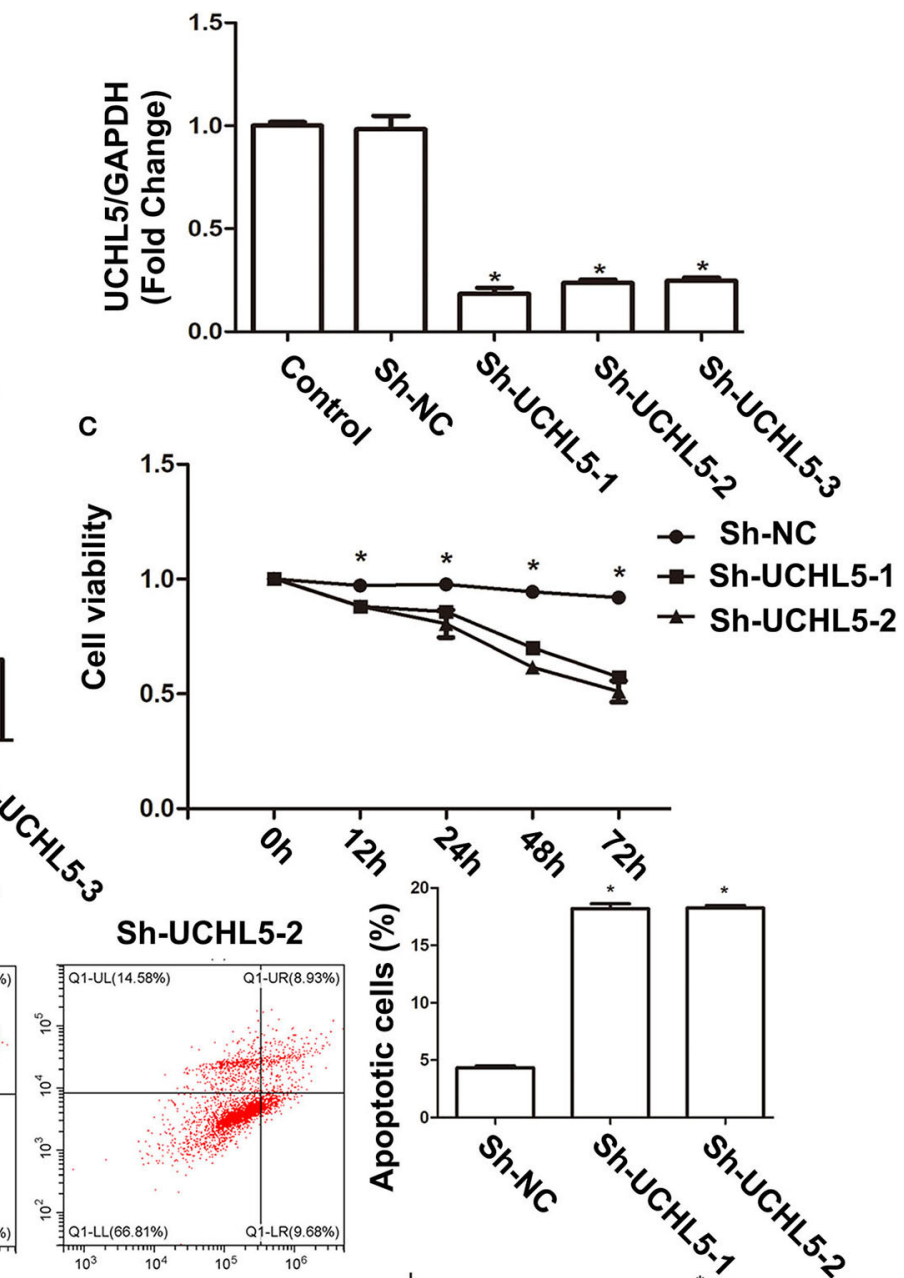

0.0
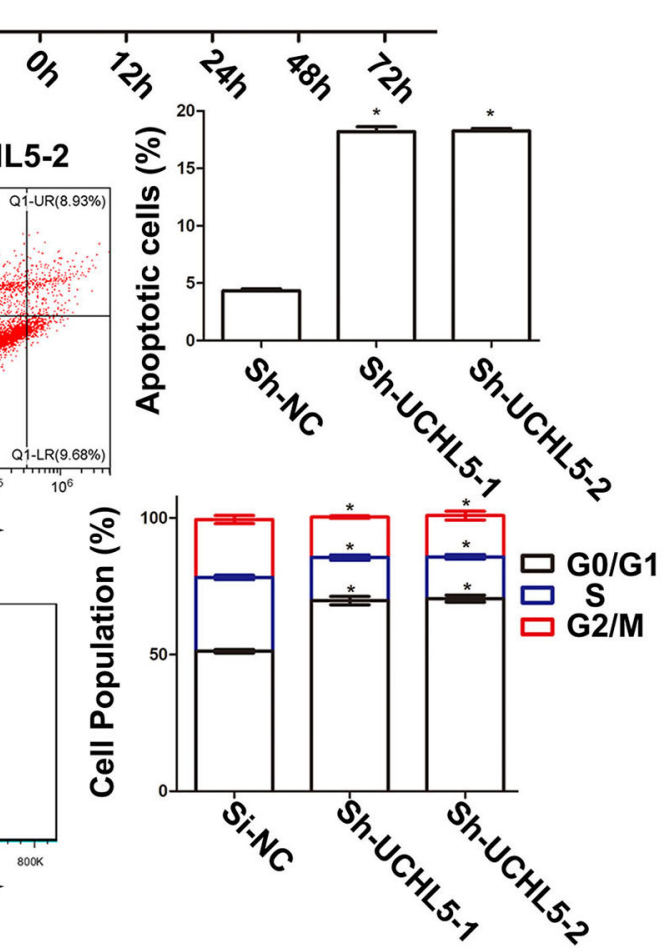

FIGURE 3 | UCHL5 knockdown decreased cell viability, increased apoptosis, and arrested cell cycle in HCE-1-A endometrial cancer cells. (A) The levels of UCHL5 were detected by WB in HCE-1-A cells at $48 \mathrm{~h}$ after shRNA lentiviral infection ( $N=3$ times). ${ }^{*}<0.05$ vs. control. (B) Relative mRNA levels of UCHL5 in shRNA lentiviral infected HCE-1-A cells $(N=3)$. ${ }^{\star} P<0.05$ vs. control. (C) Relative cell viability was detected by CCK-8 at different time points $(N=3)$. ${ }^{\star} P<0.05$ vs. sh-UCHL5-1 and sh-UCHL5-2. (D) Apoptosis cells were marked by Annexin V-FITC/PI, and the percentage was analyzed by flow cytometry in HCE-1-A cells at $48 \mathrm{~h}$ after shRNA lentiviral infection $(N \geq 3)$. ${ }^{*} P<0.05$ vs. sh-NC (negative control). (E) Cell cycle was measured by Cell Cycle Analysis Kit ( $\left.N \geq 3\right) .{ }^{\star} P<0.05$ vs. sh-NC.

\section{Wnt/ $\beta$-Catenin Signaling Pathway Inhibitor XAV939 Eliminated the Tumorigenic Effects Aroused by UCHL5 Overexpression in EC Cells}

To further confirm whether the UCHL5-regulated cell phenotype in EC cells was mediated by the Wnt/ $\beta$-catenin signaling pathway, Wnt/ $\beta$-catenin inhibitor XAV939 was used in the present study. As expected, UCHL5 overexpression induced the increase in $\beta$-catenin and its target genes CyclinD1, C-myc, and Survivin and the decrease in cleaved-caspase 3 in AN3-CA cells, which were reversed by excessive XAV939 treatment (Figures 6A-C). Additionally, XAV939 treatment showed a smaller sub-G1 population when compared with the 
A

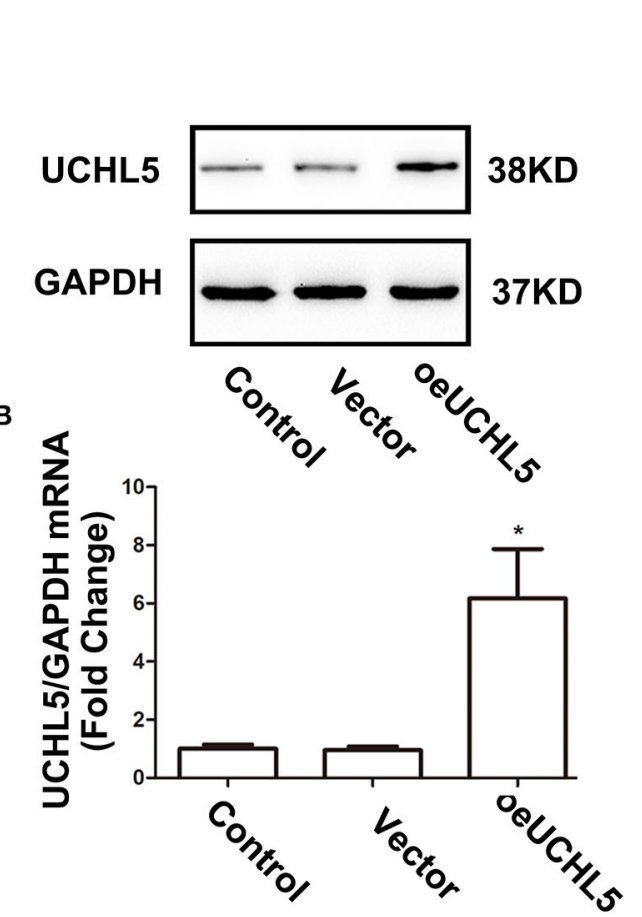

D Vector
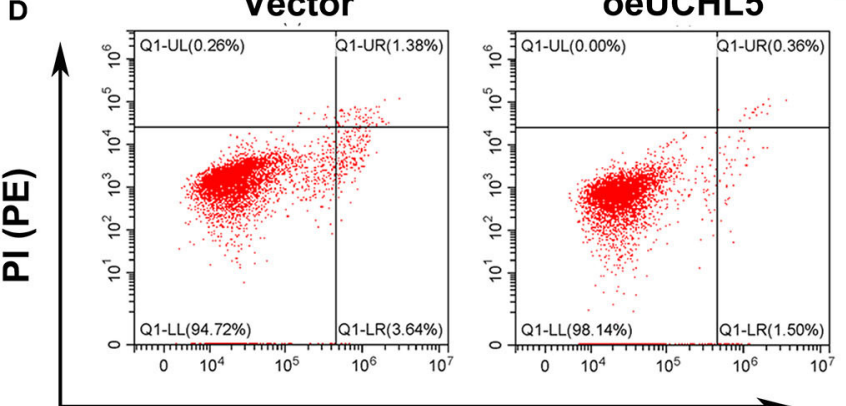

E

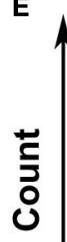

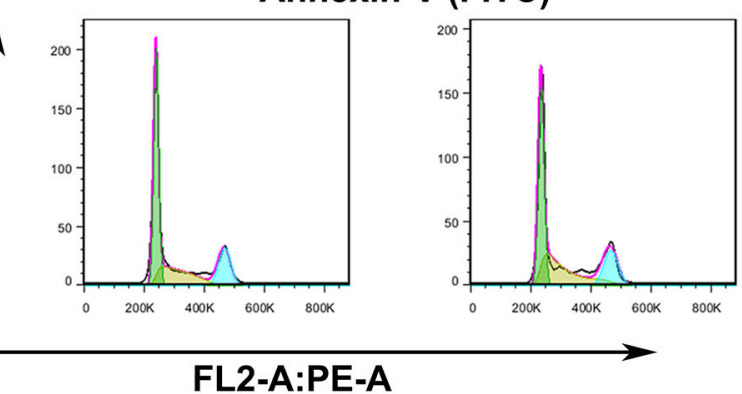

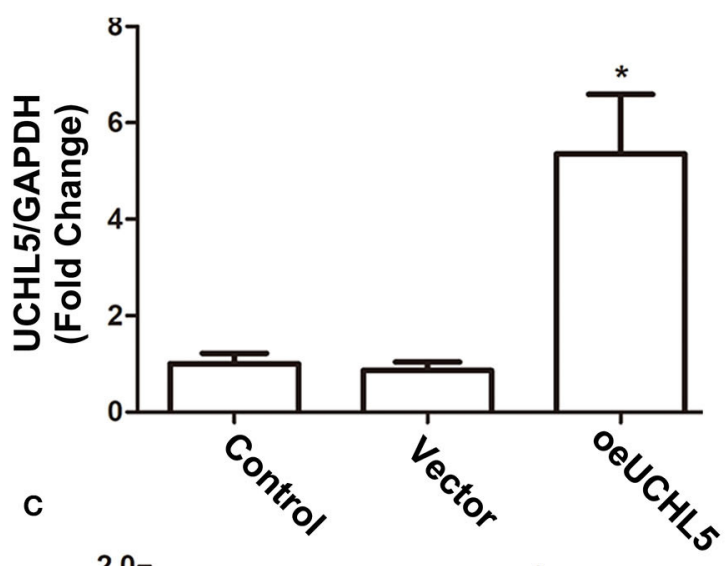

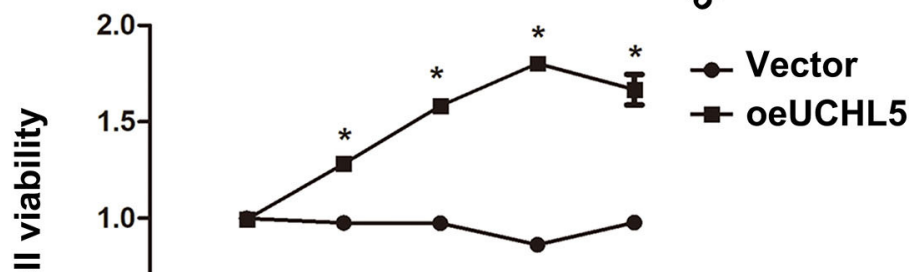

0.5

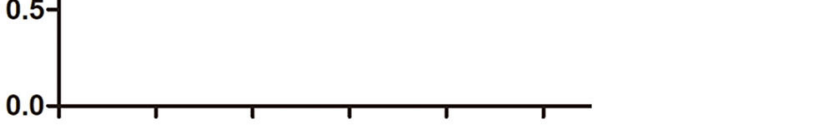


A

B
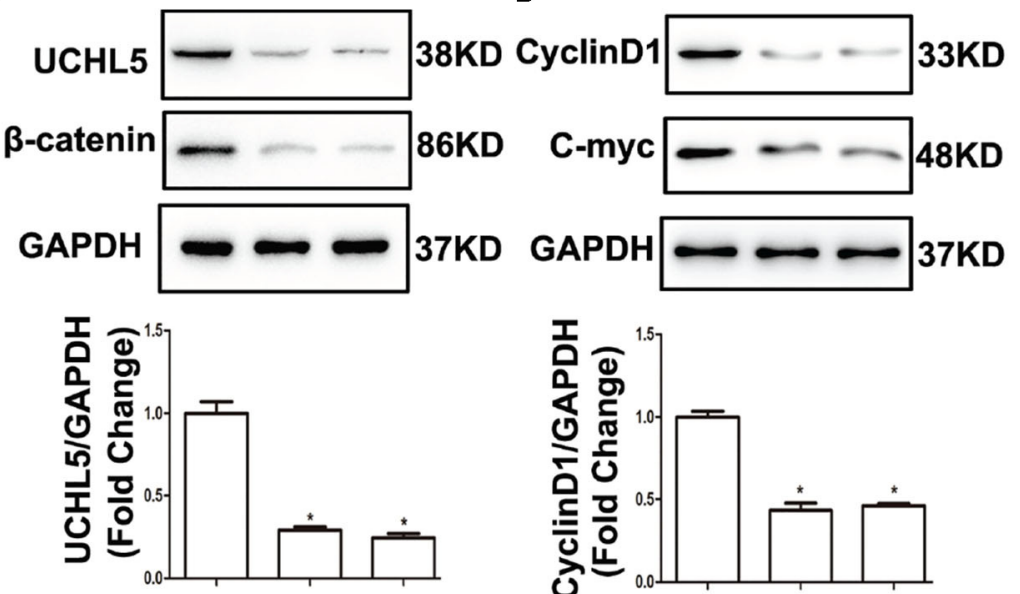

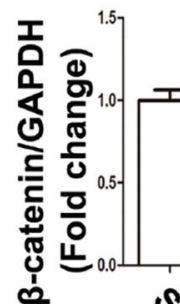

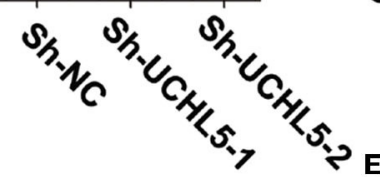

D

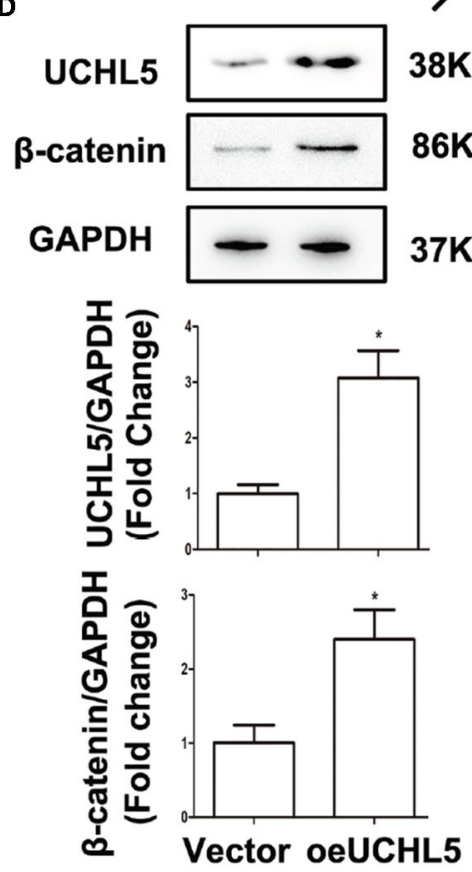

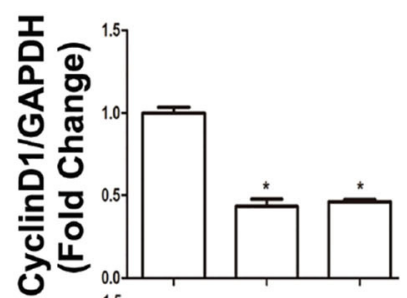

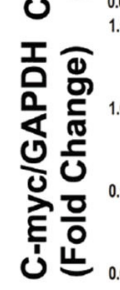

CyclinD1 $-\longrightarrow$ 33KD

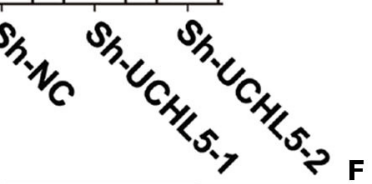

C

Cleaved-

Caspase3 $--\longrightarrow$ 32KD

Survivin $--\longrightarrow$ 16

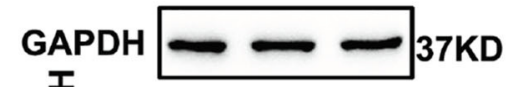

I

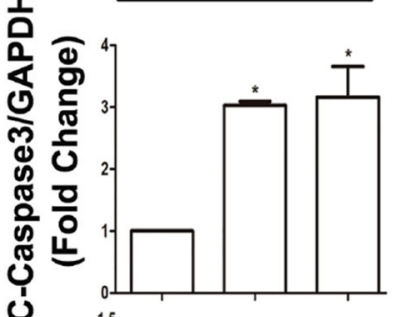

U

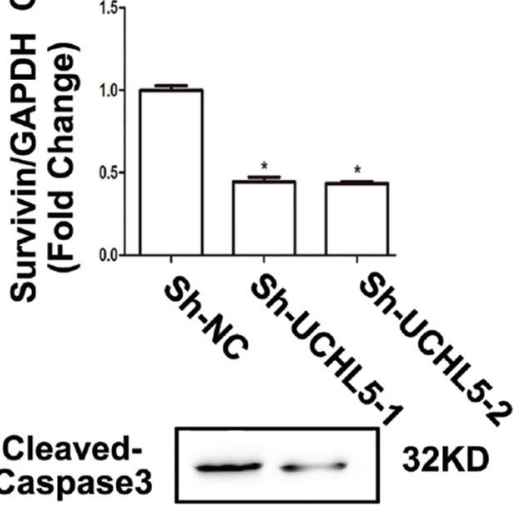

F

Caspase3

Survivin $\longrightarrow$ 16KD
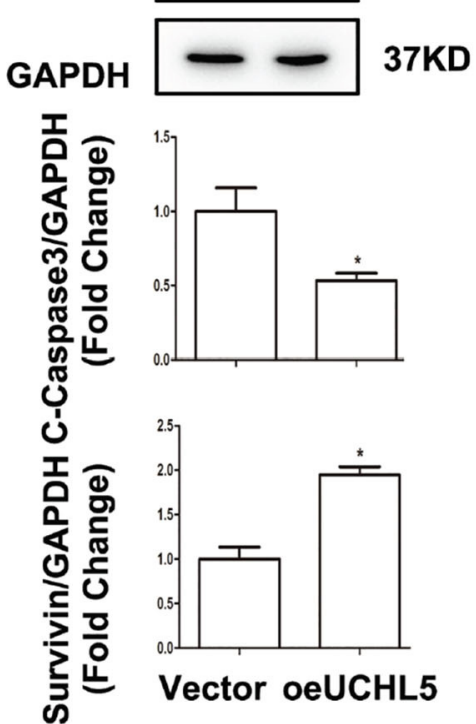

FIGURE 5 | UCHL5 activated Wnt/ $\beta$-catenin signaling and affected the expression of its target genes. (A) Relative protein levels of UCHL5 and $\beta$-catenin were detected by WB in HCE-1-A cells at $48 \mathrm{~h}$ after shRNA lentiviral infection ( $N=3$ times). ${ }^{*} P<0.05$ vs. sh-NC. (B) Relative levels of cell cycle-related protein CyclinD1 and cell proliferation-related protein C-myc. ( $N=3$ times). ${ }^{*} P<0.05$ vs. sh-NC. (C) Relative levels of cell apoptosis-related protein cleaved-caspase3 and anti-apoptosis protein Survivin ( $N=3$ times). ${ }^{*} P<0.05$ vs. sh-NC. (D) Relative protein levels of UCHL5 and $\beta$-catenin were detected by WB in AN3-CA cells at $48 \mathrm{~h}$ after infected with overexpressed lentiviral vectors ( $N=3$ times). ${ }^{*} P<0.05$ vs. empty vector. (E) Relative levels of CyclinD1 and $\mathrm{C}$-myc $\left(N=3\right.$ times). ${ }^{*} P<0.05$ vs. empty vector. (F) Relative levels of cleaved-caspase3 and Survivin ( $N=3$ times). ${ }^{*} P<0.05$ vs. empty vector. 


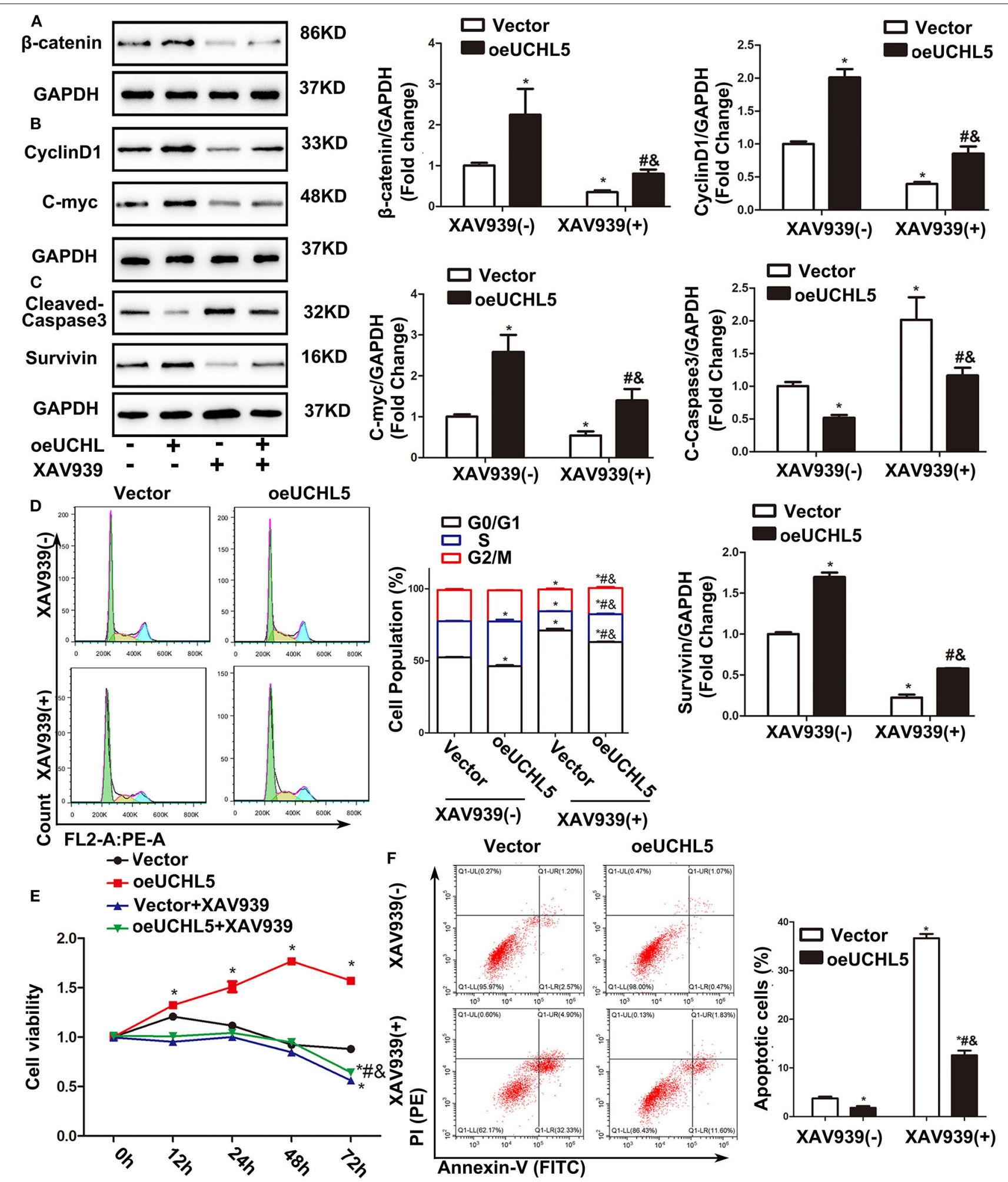

FIGURE 6 | Wnt/ $\beta$-catenin signaling pathway inhibitor XAV939 eliminated the disadvantages aroused by UCHL5 overexpression in AN3-CA cells. (A) UCHL5 overexpression (oeUCHL5). AN3-CA cells were treated with or without Wnt/ $\beta$-catenin signaling pathway inhibitor XAV939 $(10 \mu \mathrm{M})$ for $24 \mathrm{~h}$. Relative protein levels of $\beta$-catenin were detected by WB. ${ }^{*} P<0.05$ vs. empty vector; ${ }^{\#} P<0.05$ vs. oeUCHL5; ${ }^{\&} P<0.05$ vs. vector + XAV939. (B,C) Relative protein levels of CyclinD1, C-myc, cleaved-caspase3, and Survivin were detected by WB. ${ }^{*} P<0.05$ vs. empty vector; $\# P<0.05$ vs. oeUCHL5; $\& P<0.05$ vs. vector + XAV939. (D) Cell cycle was measured by Cell Cycle Analysis Kit $(N \geq 3)$. ${ }^{*} P<0.05$ vs. empty vector; ${ }^{\#} P<0.05$ vs. oeUCHL5; ${ }^{\&} P<0.05$ vs. vector + XAV939. (E) Relative cell viability were detected by CCK-8 at different time points $(N=3)$. ${ }^{*} P<0.05$ vs. empty vector; ${ }^{*} P<0.05$ vs. oeUCHL5; ${ }^{\&} P<0.05$ vs. vector $+X A V 939$. (F) The percentage of Apoptosis cells $(N \geq 3)$. ${ }^{*} P<0.05$ vs. empty vector; $\# P<0.05$ vs. oeUCHL5; ${ }^{\&} P<0.05$ vs. vector + XAV939. 


\section{UCHL5 Silence Restricted Tumor Growth via Inhibiting the Wnt/ $\beta$-Catenin Signaling Pathway in vivo}

To investigate the role of UCHL5 in tumorigenesis in vivo, the HCE-1-A cells stably transfected with lentivirus particles containing UCHL5 shRNA were injected subcutaneously into female nude mice. As shown in Figures 7A-C, UCHL5 silence obviously repressed the size, volume, and weight of the xenograft tumors in the nude mice. Hematoxylin-eosin (HE) staining of xenograft tumors revealed that UCHL5 knockdown presented better differentiated morphology and keratinization than the control group (Figure 7D). Consistent with the results aforementioned in vitro, UCHL5 silence resulted in decreases in $\beta$-catenin and its target genes CyclinD1, C-myc, and Survivin but an increase in cleaved-caspase 3 in vivo (Figures 7E-G).

In conclusion, our results that UCHL5 promoted the growth of EC in vivo and vitro via activating the $\mathrm{Wnt} / \beta$-catenin signaling pathway may provide potential targets for EC control in the future.

\section{DISCUSSION}

Considering the poor responses and prognosis of current chemotherapy drugs for EC, our enthusiasm to explore more potential anti-cancer medicine was blooming. In this study, the increase in UCHL5 expression was observed in EC tissues and negatively correlated with overall patient survival. In addition, UCHL5 overexpression mediated by lentivirus vectors activated $\mathrm{Wnt} / \beta$-catenin signaling in endometrial cancer cells and resulted in a decrease in apoptosis and an increase in proliferation. $\beta$-Catenin upregulation was the central event because of its capacity to control the expression of cell cycle checker CyclinD1, proliferation-related C-myc, apoptosis-related cleaved-caspase 3, and Survivin.

The above results were confirmed by the fact that $\mathrm{Wnt} / \beta$ catenin signaling inhibitor XAV939 eliminated the effects caused by UCHL 5 overexpression. Additionally, UCHL 5 silence mediated by sh-RNA lentivirus produced effective inhibition of EC growth in vitro and vivo. As expected, UCHL5 silence arrested cell proliferation and cycle and induced cell apoptosis via inhibiting the $\mathrm{Wnt} / \beta$-catenin signaling pathway.

Cancer cells always display high rates of protein synthesis, and the growth of tumors depends on the ubiquitin-proteasome system (UPS) for maintenance of homeostasis (10). Whatever destroyed the UPS, cancer cells would go to die for a continuous state of proteotoxic stress caused by defective protein accumulation $(16,25)$. The success of proteasome inhibitors used to treat cancer in the clinic arouses great attention on ubiquitination modulators for cancer treatment (26). DUBs prevent protein degradation by removing ubiquitin chains from protein substrates. Interestingly, increasingly more and more studies found that DUBs are overexpressed and produce promoting effects in many cancer cells (25). Moreover, a series of small molecules against DUBs have been developed and implicated in cancer treatment (27). For example, USP7 inhibitor FT671 significantly inhibited the growth of medulloblastoma, colorectal, and lung tumors in mice. Moreover, b-AP15, PtPT, and VLX1570, as inhibitors of ubiquitin-specific protease 14 (USP14) and UCHL5, induce apoptosis in myeloma, breast cancer, and prostate cancer cells $(28,29)$. Thus, DUBs seem to be excellent drug targets for cancer treatment.

UCHL5 reversibly associates with the $26 \mathrm{~S}$ proteasome and prevents target proteins degradation by hydrolyzing ubiquitin chains (30). Several studies have reported that high UCHL5 expression detected by immunohistochemistry or WB has a positive correlation with poor survival and high probability of recurrence $(15,31,32)$. However, few researches answer whether UCHL5 is expressed on human EC tissues and if UCHL5 affects their growth and long-term outcome. This study firstly reported that UCHL5 was more highly expressed on EC tissues and cell lines and UCHL5 overexpression accelerated EC growth. These results indicated that UCHL5 was an attractive target for drug development of EC treatment. It is worth emphasizing that the current inhibitors, such as b-AP15, PtPT, and VLX1570, targeting both UCHL5 and Usp-14, showed limited application for their multiple side effects. Thus, highly selective drugs targeting UCHL5 alone deserve being explored.

$\beta$-Catenin is a key signal transduction protein in the Wnt/ $\beta$-catenin pathway, which controls transcription of a wide range of genes involved in embryonic development, cell proliferation and migration, and cell fate (33). Aberrant activation of the Wnt/ $\beta$-catenin pathway has been reported in EC and associates with the deterioration outcome $(34,35)$. Previous researches documented that deletion of UCHL5 increased the level of the ubiquitinated $\beta$-catenin and accelerated the hydrogen peroxide-stimulated degradation of $\beta$-catenin in HeLa cells (36). In the study, UCHL5 overexpression elevated the levels of $\beta$-catenin and regulated its downstream genes (CyclinD1, C-myc, Survivin, and cleaved-caspase3), which were counteracted by the $\mathrm{Wnt} / \beta$-catenin inhibitor XAV939. The deeper mechanism may be that the degradation of $\beta$-catenin depends on the UCHL5-mediated ubiquitinproteasome pathway. Above all, Wnt/ $\beta$-catenin pathway activation at least partly explained the tumor-promoting effects of UCHL5.

Although this study seemed to draw a whole theory for the application of UCHL5 inhibition on EC treatment, the following limitations remain to be considered. Firstly, how was UCHL5 upregulated in cancer tissues? Three potential methylation sites in the UCHL5 promoter have been predicted in previous studies (37). However, more methylation sites and microRNAs may be important breakthroughs. The deubiquitination activity of UCHL5 depends on the recruitment by hRpn13 which is a component of the $19 \mathrm{~S}$ particle and bind to UCHL5 via KEKE motifs. Thus, the levels of hRpn13 and its regulating effects on UCHL5 in EC should be detected in future studies. Additionally, previous research believed that nuclear or cytoplasmic UCHL5 expression, respectively, was reported to be a predictor for survival in several cancers $(38,39)$. However, the difference of the expression and function between cytoplasmic and nucleus UCHL5 was not compared in current known studies. 


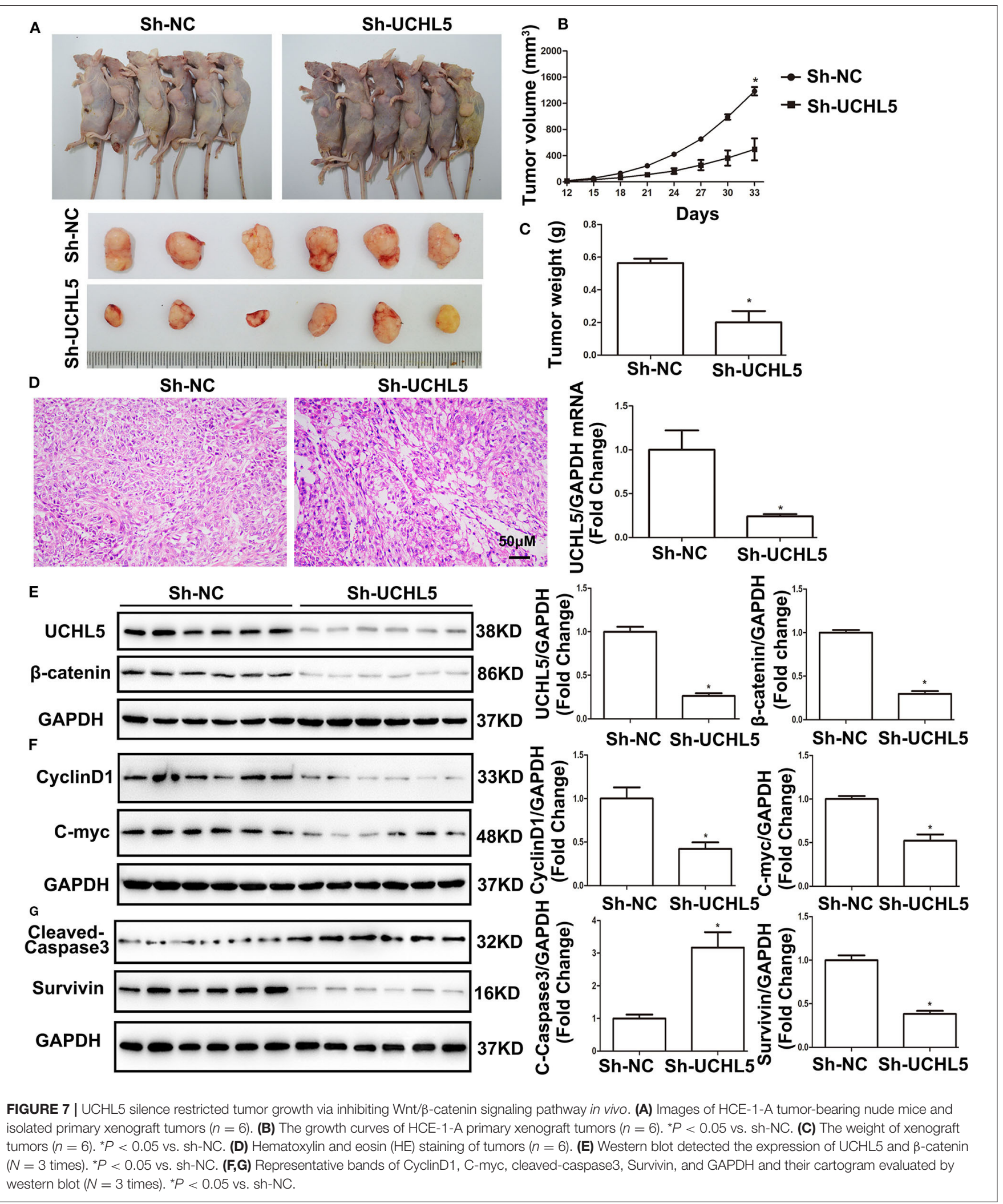

In summary, this study provides new insights into the effects of the deubiquitinating enzyme UCHL5 in EC. UCHL5 not only overexpressed on EC tissues and cell lines but also promoted their growth. We suspect that it is because UCHL5 rescues the ubiquitination degradation of $\beta$-catenin. The elevated $\beta$ catenin stimulated cell proliferation, accelerated cell cycle, and 
decreased apoptosis via regulating its downstream proteins in EC cells. Thus, UCHL5 plays an important role in EC growth and is hopefully developed into a new therapeutic strategy for EC.

\section{DATA AVAILABILITY STATEMENT}

The datasets generated for this study are available on request to the corresponding author.

\section{ETHICS STATEMENT}

The studies involving human participants were reviewed and approved by Ethics Committee of Shengjing Hospital affiliated with China Medical University. The patients/participants

\section{REFERENCES}

1. Siegel RL, Miller KD, Jemal A. Cancer statistics, 2019. CA Cancer J Clin. (2019) 69:7-34. doi: 10.3322/caac.21551

2. Onstad MA, Schmandt RE, Lu KH. Addressing the role of obesity in endometrial cancer risk, prevention, and treatment. J Clin Oncol. (2016) 34:4225-30. doi: 10.1200/JCO.2016.69.4638

3. von Gruenigen VE, Tian C, Frasure H, Waggoner S, Keys H, Barakat RR. Treatment effects, disease recurrence, and survival in obese women with early endometrial carcinoma : a Gynecologic Oncology Group study. Cancer. (2006) 107:2786-91. doi: 10.1002/cncr.22351

4. Sehouli J, Koensgen D, Oskay-Ozcelik G, Mustea A. New aspects of adjuvant therapy in endometrial cancer: current standards and future directions. Crit Rev Oncol Hematol. (2008) 67:204-12. doi: 10.1016/j.critrevonc.2008.02.011

5. de Boer SM, Powell ME, Mileshkin L, Katsaros D, Bessette P, Haie-Meder $\mathrm{C}$, et al. Adjuvant chemoradiotherapy versus radiotherapy alone in women with high-risk endometrial cancer (PORTEC-3): patterns of recurrence and post-hoc survival analysis of a randomised phase 3 trial. Lancet Oncol. (2019) 20:1273-85. doi: 10.1016/S1470-2045(19)30395-X

6. Ciechanover A. The ubiquitin proteolytic system: from a vague idea, through basic mechanisms, and onto human diseases and drug targeting. Neurology. (2006) 66:S7-19. doi: 10.1212/01.wnl.0000192261.02023.b8

7. Bader N, Jung $\mathrm{T}$, Grune $\mathrm{T}$. The proteasome and its role in nuclear protein maintenance. Exp Gerontol. (2007) 42:864-70. doi: 10.1016/j.exger.2007.03.010

8. Lecker SH, Goldberg AL, Mitch WE. Protein degradation by the ubiquitinproteasome pathway in normal and disease states. J Am Soc Nephrol. (2006) 17:1807-19. doi: 10.1681/ASN.2006010083

9. Welchman RL, Gordon C, Mayer RJ. Ubiquitin and ubiquitin-like proteins as multifunctional signals. Nat Rev Mol Cell Biol. (2005) 6:599-609. doi: $10.1038 / \mathrm{nrm} 1700$

10. D'Arcy P, Linder S. Proteasome deubiquitinases as novel targets for cancer therapy. Int J Biochem Cell Biol. (2012) 44:1729-38. doi: 10.1016/j.biocel.2012.07.011

11. Huang OW, Cochran AG. Regulation of deubiquitinase proteolytic activity. Curr Opin Struct Biol. (2013) 23:806-11. doi: 10.1016/j.sbi.2013.07.012

12. Brnjic S, Mazurkiewicz M, Fryknäs M, Sun C, Zhang X, Larsson R, et al. Induction of tumor cell apoptosis by a proteasome deubiquitinase inhibitor is associated with oxidative stress. Antioxid Redox Signal. (2014) 21:2271-85. doi: 10.1089/ars.2013.5322

13. Chen X, Walters KJ. Structural plasticity allows UCH37 to be primed by RPN13 or locked down by INO80G. Mol Cell. (2015) 57:767-8. doi: 10.1016/j.molcel.2015.02.025

14. Al-Shami A, Jhaver KG, Vogel P, Wilkins C, Humphries J, Davis $\mathrm{JJ}$, et al. Regulators of the proteasome pathway, Uch37 and Rpn13, provided their written informed consent to participate in this study. The animal study was reviewed and approved by Ethics Committee of Shengjing Hospital affiliated with China Medical University.

\section{AUTHOR CONTRIBUTIONS}

DL and LO developed the hypothesis, designed the experiments, and wrote this manuscript. DL, ZS, and XW performed the experiments and analyzed the data.

\section{FUNDING}

This study was supported by Liaoning Scientific Research Funding Project (JC2019012) and Shenyang Science and Technology Plan Project (19-112-4-020).

play distinct roles in mouse development. PLoS One. (2010) 5:e13654. doi: 10.1371/journal.pone.0013654

15. Wang L, Chen YJ, Xu K, Wang YY, Shen XZ, Tu RQ. High expression of $\mathrm{UCH} 37$ is significantly associated with poor prognosis in human epithelial ovarian cancer. Tumour Biol. (2014) 35:11427-33. doi: 10.1007/s13277-014-2446-3

16. D'Arcy P, Linder S. Molecular pathways: translational potential of deubiquitinases as drug targets. Clin Cancer Res. (2014) 20:3908-14. doi: 10.1158/1078-0432.CCR-14-0568

17. MacDonald BT, Tamai $\mathrm{K}$, He $\mathrm{X}$. Wnt $/ \beta$-catenin signaling: components, mechanisms, and diseases. Dev Cell. (2009) 17:9-26. doi: 10.1016/j.devcel.2009.06.016

18. Kotrbová A, Ovesná $\mathrm{P}$, Gybel' T, Radaszkiewicz T, Bednaríková M, Hausnerová J, et al. WNT signaling inducing activity in ascites predicts poor outcome in ovarian cancer. Theranostics. (2020) 10:537-52. doi: $10.7150 /$ thno. 37423

19. Wu X, Xia T, Cao M, Zhang P, Shi G, Chen L, et al. LncRNA BANCR promotes pancreatic cancer tumorigenesis via modulating MiR-195-5p/Wnt/ $\beta$-catenin signaling pathway. Technol Cancer Res Treat. (2019) 18:1533033819887962. doi: $10.1177 / 1533033819887962$

20. Shao TR, Zheng ZN, Chen YC, Wu QQ, Huang GZ, Li F, et al. LncRNA AC007271.3 promotes cell proliferation, invasion, migration and inhibits cell apoptosis of OSCC via the Wnt/ $\beta$-catenin signaling pathway. Life Sci. (2019) 239:117087. doi: 10.1016/j.lfs.2019.117087

21. Lois C, Hong EJ, Pease S, Brown EJ, Baltimore D. Germline transmission and tissue-specific expression of transgenes delivered by lentiviral vectors. Science. (2002) 295:868-72. doi: 10.1126/science.1067081

22. Dull T, Zufferey R, Kelly M, Mandel RJ, Nguyen M, Trono D, et al. A thirdgeneration lentivirus vector with a conditional packaging system. J Virol. (1998) 72:8463-71. doi: 10.1128/JVI.72.11.8463-8471.1998

23. Wu L, Wang W, Dai M, Li H, Chen C, Wang D. PPAR $\alpha$ ligand, AVE8134, and cyclooxygenase inhibitor therapy synergistically suppress lung cancer growth and metastasis. BMC Cancer. (2019) 19:1166. doi: 10.1186/s12885-019-6379-5

24. Saxena V, Orgill D, Kohane I. Absolute enrichment: gene set enrichment analysis for homeostatic systems. Nucleic Acids Res. (2006) 34:e151. doi: $10.1093 /$ nar/gkl766

25. Selvaraju K, Mazurkiewicz M, Wang X, Gullbo J, Linder S, D’Arcy P. Inhibition of proteasome deubiquitinase activity: a strategy to overcome resistance to conventional proteasome inhibitors? Drug Resist Updat. 2015:209. doi: 10.1016/j.drup.2015.06.001

26. Teicher BA, Tomaszewski JE. Proteasome inhibitors. Biochem Pharmacol. (2015) 96:1-9. doi: 10.1016/j.bcp.2015.04.008

27. Harrigan JA, Jacq X, Martin NM, Jackson SP. Deubiquitylating enzymes and drug discovery: emerging opportunities. Nat Rev Drug Discov. (2018) 17:57-78. doi: 10.1038/nrd.2017.152 
28. Fukui S, Nagasaka K, Miyagawa Y, Kikuchi-Koike R, Kawata Y, Kanda $\mathrm{R}$, et al. The proteasome deubiquitinase inhibitor bAP15 downregulates TGF- $\beta /$ Smad signaling and induces apoptosis via UCHL5 inhibition in ovarian cancer. Oncotarget. (2019) 10:5932-48. doi: 10.18632/oncotarget. 27219

29. Paulus A, Akhtar S, Caulfield TR, Samuel K, Yousaf H, Bashir Y, et al. Coinhibition of the deubiquitinating enzymes, USP14 and UCHL5, with VLX1570 is lethal to ibrutinib- or bortezomib-resistant Waldenstrom macroglobulinemia tumor cells. Blood Cancer J. (2016) 6:e492. doi: $10.1038 /$ bcj.2016.93

30. Vander Linden RT, Hemmis CW, Schmitt B, Ndoja A, Whitby FG, Robinson $\mathrm{H}$, et al. Structural basis for the activation and inhibition of the UCH37 deubiquitylase. Mol Cell. (2015) 57:901-11. doi: 10.1016/j.molcel.2015.01.016

31. Chen Y, Fu D, Xi J, Ji Z, Liu T, Ma Y, et al. Expression and clinical significance of UCH37 in human esophageal squamous cell carcinoma. Dig Dis Sci. (2012) 57:2310-7. doi: 10.1007/s10620-012-2181-9

32. Chen YJ, Ma YS, Fang Y, Wang Y, Fu D, Shen XZ. Power and promise of ubiquitin carboxyl-terminal hydrolase 37 as a target of cancer therapy. Asian Pac J Cancer Prev. (2013) 14:2173-9. doi: 10.7314/APJCP.2013.14. 4.2173

33. Clevers H, Nusse R. Wnt/ $\beta$-catenin signaling and disease. Cell. (2012) 149:1192-205. doi: 10.1016/j.cell.2012.05.012

34. Lv Y, Zhang L, Ma J, Fei X, Xu K, Lin J. CTHRC1 overexpression promotes ectopic endometrial stromal cell proliferation, migration and invasion via activation of the Wnt/ $\beta$-catenin pathway. Reprod Biomed Online. (2019) 40:26-32. doi: 10.1016/j.rbmo.2019.10.001

35. Huang $X$, Zhong $\mathrm{R}, \mathrm{He} X$, Deng Q, Peng $\mathrm{X}, \mathrm{Li}$, et al. Investigations on the mechanism of progesterone in inhibiting endometrial cancer cell cycle and viability via regulation of long noncoding RNA NEAT1/microRNA- 146b-5p mediated Wnt/ $\beta$-catenin signaling. IUBMB Life. (2019) 71:223-34. doi: 10.1002/iub.1959

36. Li Z, Zhou L, Jiang T, Fan L, Liu X, Qiu X. Proteasomal deubiquitinase $\mathrm{UCH} 37$ inhibits degradation of $\beta$-catenin and promotes cell proliferation and motility. Acta Biochim Biophys Sin. (Shanghai). (2019) 51:277-84. doi: 10.1093/abbs/gmy176

37. Jiao L, Ouyang S, Shaw N, Song G, Feng Y, Niu F, et al. Mechanism of the Rpn13-induced activation of Uch37. Protein Cell. (2014) 5:616-30. doi: 10.1007/s13238-014-0046-Z

38. Arpalahti L, Saukkonen K, Hagström J, Mustonen H, Seppänen H, Haglund C, et al. Nuclear ubiquitin C-terminal hydrolase L5 expression associates with increased patient survival in pancreatic ductal adenocarcinoma. Tumour Biol. (2017) 39:1010428317710411. doi: 10.1177/1010428317710411

39. Arpalahti L, Laitinen A, Hagström J, Mustonen H, Kokkola A, Böckelman C, et al. Positive cytoplasmic UCHL5 tumor expression in gastric cancer is linked to improved prognosis. PLoS One. (2018) 13:e0193125. doi: 10.1371/journal.pone.0193125

Conflict of Interest: The authors declare that the research was conducted in the absence of any commercial or financial relationships that could be construed as a potential conflict of interest.

Copyright (C) 2020 Liu, Song, Wang and Ouyang. This is an open-access article distributed under the terms of the Creative Commons Attribution License (CC BY). The use, distribution or reproduction in other forums is permitted, provided the original author(s) and the copyright owner(s) are credited and that the original publication in this journal is cited, in accordance with accepted academic practice. No use, distribution or reproduction is permitted which does not comply with these terms. 